\title{
Asynchronous Adaptive Fault-Tolerant Control for Markov Jump Systems with Actuator Failures and Unknown Nonlinear Disturbances
}

\author{
Jian-Ning Li (D), Kai-Yang Gu, Xiao Liu, and Xiao-Bin Xu $\mathbb{D}$ \\ Institute of System Science and Control Engineering, School of Automation, Hangzhou Dianzi University, \\ Hangzhou 310018, China \\ Correspondence should be addressed to Jian-Ning Li; ljn@hdu.edu.cn
}

Received 29 October 2019; Revised 17 December 2019; Accepted 5 February 2020; Published 9 March 2020

Academic Editor: Mondher Farza

Copyright (c) 2020 Jian-Ning Li et al. This is an open access article distributed under the Creative Commons Attribution License, which permits unrestricted use, distribution, and reproduction in any medium, provided the original work is properly cited.

This article investigates an asynchronous adaptive fault-tolerant control problem for Markov jump systems (MJSs) with actuator failures and absolutely unknown nonlinear disturbances. The hidden Markov model (HMM) is used to describe the asynchronization phenomenon between the controller and the modes of the original system. Moreover, by using the designed adaptive law, the values of actuator partial failures and the bounds of the unknown nonlinear disturbances can be estimated, respectively. Furthermore, the HMM-based asynchronous adaptive fault-tolerant controller is provided to ensure that the closed-loop system is asymptotically stable despite of unknown nonlinear disturbances. Finally, numerical examples are given to show the effectiveness of the proposed method.

\section{Introduction}

MJS is a special hybrid system with a wide range of applications, such as chemical plant, aircrafts, and robotic systems. In such systems, the loss of components after years of disrepair, the system parameters, and structural changes which are caused by sudden external environment changes can be described as the Markov process. In the past few decades, based on the characteristic and its remarkable modeling capabilities, the MJS has received considerable attractions and a number of results have been published. For instance, the stability and controllability of the MJS are studied in [1-5]. Afterwards, more in-depth research studies of the MJS were carried out, such as $H_{\infty}$ control, robust fault-tolerant control, guaranteed cost control, and sliding mode control [6-10].

In the recent period of time, the plant modes are not always accessible due to the loss of data, quantization, and time delay, so the design of the asynchronous controller/ filter has received a lot of attentions. The asynchronous $L_{2}-$ $L_{\infty}$ filtering problem for discrete-time random MJS with sensor nonlinearities is studied in [11], and it is assumed that the sensor nonlinearity occurs randomly satisfying the Bernoulli distribution. In [12], the distributed $H_{\infty}$ filtering problem for a class of discrete-time Markov jump Lur'e systems with a stochastic switching topology is studied. A new class of distributed filters is designed to reflect the target plants with the mismatched characteristic of modes jumping between the target plants and the proposed filters, and it makes the filtering error system stochastically stable. Another nonsynchronous mechanism is introduced in [13], that is, HMM. The problem of the random passive asynchronous control is studied in [14] for MJS, and an asynchronous controller is designed. At present, there are many achievements in the research of the controller with switching asynchronous problems caused by time delay. In [15], a robust reliable controller is designed for a class of nonlinear switching systems with time delay and actuator failures. The closed-loop control system under asynchronous switching can be exponentially stable. In the case of the switching system with matched or mismatched condition, the scheme of segmentation Lyapunov function and average dwell time 
is proposed in [16] to solve the problem of hysteresis between the switching of filters and subsystems. In the actual industrial system, it is inevitable that the controller will not be matched with the system. This makes the asynchronization of controllers and systems become a common phenomenon, and it is one motivation of this paper.

In most control systems, the occurrence of failures is uncertain in time and the value of failures is also unknown. Failures can cause the performance of systems to drop or crash directly. Therefore, in the past decades, designing faulttolerant controllers to ensure system stability and performance indicators is also one of the hot spots. The design method of the fault-tolerant controller can mainly be divided into two categories: passive fault-tolerant control [17-20] and active fault-tolerant control [21-23]. The passive fault-tolerant control algorithm is designing a controller with a fixed gain by using the robust control method, but the result is conservative. The active fault-tolerant control approach compensates for the failures by reconstructing a new controller online with forming a suitable fault-tolerant control law. Adaptive fault-tolerant control is a popular branch of the active fault-tolerant control $[11,25]$, and it has yielded many results. In references [26, 27], the authors have proposed an adaptive state-feedback controller for the systems with actuator failures. The adaptive controllers of Markov and stochastic systems are provided in [28, 29]. Recently, some research studies on the asynchronous fault-tolerant controller have been provided in $[30,31]$. However, the considered disturbance in these research studies should satisfy some assumptions in advance, such as satisfies Wiener process or belongs to $L_{2}[0,+\infty]$. In practice, the stability problem for systems with unknown nonlinear disturbance is much more complicated, which is the second motivation of this research.

The purpose of this paper is to design an asynchronous adaptive fault-tolerant controller for MJSs with actuator failures and unknown nonlinear disturbances. The asynchronization phenomenon between the controller modes and the system modes is described by the HMM. Then, an adaptive fault-tolerant control method is proposed for the MJS with actuator failures and unknown nonlinear disturbances. The corresponding values of the partial failures and the upper and lower bounds of unknown nonlinear disturbance are estimated via the adaptive laws. Furthermore, the adaptive fault-tolerant controller is provided based on the estimated results and the effects of failures and nonlinear disturbances can be compensated automatically. By utilizing the Lyapunov stability theory, the stability of the closed-loop system can be guaranteed in the presence of failures and unknown nonlinear disturbances. The main contributions of this paper can be summarised as follows: (1) for a faulty MJS with unknown nonlinear disturbance, a novel asynchronous adaptive fault-tolerant controller is provided. (2) Combined adaptive algorithm together with the robust feedback controller only needs to use the estimation information of unknown disturbances bound, and the obtained algorithm is much simpler than the traditional adaptive control method for systems with unknown nonlinear disturbances. The rest of this paper is organized as follows. The establishment of the model is described in Section 2. In Section 3, an adaptive fault-tolerant controller is designed to solve the asynchronous control problem of MJSs. Two numerical examples are given in Section 4 to verify the effectiveness of the method. Eventually, there is a conclusion in Section 5.

\section{Preliminaries}

In this section, a kind of nonlinear MJSs is considered and the model is described as follows:

$$
\dot{x}(t)=A(r(t)) x(t)+W(r(t)) \omega(t)+B(r(t)) u^{A}(t),
$$

where $x(t) \in R^{n}$ is the system state vector, $u^{A}(t) \in R^{m}$ is the asynchronous control input vector from the actuator to the controlled object, $\omega(t) \in R^{q}$ is the external nonlinear disturbance which is assumed to be bounded but unknown, and $A(r(t)), B(r(t))$, and $W(r(t))$ are the system parameter matrices with appropriate dimensions. The variable $r(t) \in \mathcal{N}=\{1,2, \ldots, N\}$ represents the time-homogeneous Markov jump with the right continuous trajectories, and $\sigma(t) \in \mathscr{M}=\{1,2, \ldots, M\}$ represents the controller mode.

The transition probability matrix of $r(t)$ of the system model is defined as $\Pi=\left\{\pi_{i j}\right\}$

$$
\operatorname{Pr}\{r(t+\Delta)=j \mid r(t)=i\}= \begin{cases}\pi_{i j} \Delta+\mathrm{O}(\Delta), & i \neq j, \\ 1+\pi_{i j} \Delta+\mathrm{O}(\Delta), & i=j,\end{cases}
$$

where $\Delta>0, \lim _{\Delta \longrightarrow 0}(\mathrm{O}(\Delta)) / \Delta=0$, and $\pi_{i j}$ denotes the jump probability from mode $i$ at time $t$ to mode $j$ at time $t+\Delta, \pi_{i j} \geq 0$, for $i \neq j$, and $\sum_{j=1, i \neq j}^{N} \pi_{i j}=-\pi_{i i}, i=1, \ldots, N$. Considering the partial failure of the actuator,

$$
u^{A}(t)=\rho u(t)
$$

where $\rho=\operatorname{diag}\left\{\rho_{1}, \rho_{2}, \ldots, \rho_{m}\right\}$ and the partial failure of the actuator $\rho_{h}$ is an unknown constant satisfying $0<\rho_{h} \leq \rho_{h} \leq \bar{\rho}_{h} \leq 1, h=1,2, \ldots, m$. When $\rho_{h}=1$, it indicates that the $h^{\text {th }}$ actuator is operating normally; when $0<\rho_{h}<1$, it means that the $h^{\text {th }}$ actuator has occurred partial failure. The new system model can be rewritten into the following form:

$$
\dot{x}(t)=A(r(t)) x(t)+B(r(t)) \rho u(t)+W(r(t)) \omega(t) .
$$

The asynchronous fault-tolerant controller is designed by the adaptive scheme and the state-feedback approach, as follows:

$$
\begin{aligned}
u(t)= & K_{1}(\sigma(t)) x(t)+\widehat{\rho}^{-1}(t) K_{2}(\sigma(t)) x(t) \\
& +K_{3}(\sigma(t))\left[\left(I-\tau_{\omega}\right) \widehat{\bar{\omega}}(t)+\tau_{\omega} \widehat{\widehat{\omega}}(t)\right]
\end{aligned}
$$

where $K_{1}(\sigma(t)), K_{2}(\sigma(t))$, and $K_{3}(\sigma(t))$ are the controller parameters to be designed and $\hat{\rho}(t), \widehat{\bar{\omega}}(t)$, and $\underline{\widehat{\omega}}(t)$ are the estimated values of the unknown partial failure $\rho$ and the estimated values of the upper and lower bounds of the unknown nonlinear disturbance $\omega(t)$, respectively.

$$
\begin{aligned}
\tau_{\omega} & =\operatorname{diag}\left\{\tau_{\omega 1}, \ldots, \tau_{\omega q}\right\}, \\
\tau_{\omega k} & = \begin{cases}0, & \delta \zeta_{k} \geq 0, \\
1, & \delta \zeta_{k}<0 .\end{cases}
\end{aligned}
$$


The parameters $\delta, \zeta_{k}$ will be introduced later. The controller jump probability matrix is defined as $\boldsymbol{\Omega}=\left\{\mu_{i \phi}\right\}$

$$
\operatorname{Pr}\{\sigma(t)=\phi \mid r(t)=i\}=\left\{\mu_{i \phi}\right\},
$$

where the conditional probability $\mu_{i \phi} \geq 0$ and $\sum_{\phi=1}^{M} \mu_{i \phi}=1$. For simplicity, denoting $\sigma(t)=\phi$, we have

$$
\begin{aligned}
u(t)= & K_{1 \phi} x(t)+\widehat{\rho}^{-1}(t) K_{2 \phi} x(t) \\
& +K_{3 \phi}\left[\left(I-\tau_{\omega}\right) \widehat{\bar{\omega}}(t)+\tau_{\omega} \underline{\widehat{\omega}}(t)\right] .
\end{aligned}
$$

Remark 1. The stochastic process $r(t)$ in equation (2) is used to describe the system mode; however, in practice, such information is hard to obtain directly for the controller due to some unreliable condition, as packet dropouts and quantization $[11,13,32]$. The controller mode is denoted as $\sigma(t)$, and by constructing a hidden Markov model $(r(t), \sigma(t), \Pi, \boldsymbol{\Omega})$, we have (1) when the controller has only one mode, that is, $\mathscr{M}=\{1\}$, it becomes a modeindependent controller. (2) The synchronous controller is in the case of $\mathscr{M}=\mathcal{N}$ and $\mu_{i \phi}=1$ with $i=\phi$. (3) The rest of the situation is an asynchronous controller.

In order to better carry out the next research, the following assumptions are necessary.

Assumption 1. All states of the system are readily measurable.

Assumption 2. $\left\{A_{i}, B_{i} \rho\right\}$ is completely controllable for any actuator failure $\rho=\operatorname{diag}\left\{\rho_{1}, \rho_{2}, \ldots, \rho_{h}\right\}$.

Assumption 3. For system (4), in order to make the disturbance and input signal satisfy the match condition, there are constant matrices $F_{i}$ with appropriate dimensions such that

$$
W_{i}=B_{i} F_{i} \text {. }
$$

Assumption 4. For any actuator failure $\rho=\operatorname{diag}\left\{\rho_{1}, \rho_{2}, \ldots\right.$, $\left.\rho_{h}\right\}, \operatorname{rank}\left[B_{i} \rho\right]=\operatorname{rank}\left[B_{i}\right]$.

Remark 2. It is no doubt that Assumption 1 is the standard for the state-feedback system design. Assumption 2 is also a standard for the state-feedback system design, and it denotes the internal stabilizability of each normal and fault isolated system. Assumption 3 is a match condition of the disturbance and the input signal which means disturbance and input signal share the same input channel. A condition of the system with actuator redundancy is introduced in Assumption 4.

\section{Main Results}

In this section, we develop the adaptive laws to estimate the unknown partial failure $\rho$, which is denoted as $\hat{\rho}(t)$, and the upper and lower bounds of unknown nonlinear disturbances $\omega(t)$, as $\widehat{\bar{\omega}}(t), \underline{\hat{\omega}}(t)$. Then, an adaptive fault-tolerant asynchronous controller is designed to ensure that the closedloop system is asymptotically stable.

Combining with (4), (8), and (9), the closed-loop system can be described as the following equation:

$$
\begin{aligned}
\dot{x}(t)= & \left(A_{i}+B_{i} \rho K_{1 \phi}+B_{i} \rho \widehat{\rho}^{-1}(t) K_{2 \phi}\right) x(t) \\
& +B_{i} \rho K_{3 \phi}\left[\left(I-\tau_{\omega}\right) \widehat{\bar{\omega}}(t)+\tau_{\omega} \underline{\widehat{\omega}}(t)\right]+B_{i} F_{i} \omega(t) .
\end{aligned}
$$

The adaptive law of failure is designed as follows:

$$
\begin{aligned}
& \dot{\hat{\rho}}_{h}(t) \triangleq \operatorname{Proj}\left[\min \left\{\rho_{h}\right\}, \max \left\{\rho_{h}\right\}\right]\left[L_{h}(t)\right\} \\
& = \begin{cases}0, & \text { if } \hat{\rho}_{h}=\min \left\{\underline{\rho}_{h}\right\}, \text { and } L_{h} \leq 0, \\
& \text { or } \hat{\rho}_{h}=\max \left\{\bar{\rho}_{h}\right\}, \text { and } L_{h} \geq 0, \\
L_{h}(t), & \text { otherwise, }\end{cases}
\end{aligned}
$$

where $\quad L_{h}(t)=-\gamma_{h} l_{h}\left[-\hat{\rho}_{h}^{-1}(t) \delta_{h}^{2}+\hat{\rho}_{h}^{-1}(t) \delta_{h} S_{h}\right], \quad \gamma_{h}=$ $\operatorname{sign}\left(\zeta_{k h} \delta_{h}\right)$, and $\zeta_{k h}$ is the $h^{\text {th }}$ element of $\zeta_{k}$. The adaptive laws for the upper and lower bounds of nonlinear disturbances are designed as follows:

$$
\begin{aligned}
& \dot{\hat{\bar{\omega}}}_{k}(t)=\eta_{k}\left(1-\tau_{\omega k}\right) \delta \zeta_{k}, \\
& \underline{\hat{\omega}}_{k}(t)=\eta_{k} \tau_{\omega k} \delta \zeta_{k},
\end{aligned}
$$

where $\widehat{\bar{\omega}}_{k}$ and $\widehat{\omega}_{k}$ are the estimations of the upper and lower bounds of the nonlinear disturbance, $\widehat{\rho}_{h}$ is the estimation of the partial failure, $l_{h}>0$ and $\eta_{k}>0$ are the adaptive law gain for the specific application, and $\delta_{h}$ and $S_{h}$ are the $h^{\text {th }}$ component of $\delta$ and $S$, respectively:

$$
\begin{aligned}
\delta & =\left[\max \left\{x^{T}(t) P_{d} \operatorname{col}_{1}\left(B_{d}\right)\right\}, \ldots, \max \left\{x^{T}(t) P_{d} \operatorname{col}_{m}\left(B_{d}\right)\right\}\right], \\
\zeta_{k} & =\left[\max \left\{\left(F_{d}\right)_{1 k}\right\}, \max \left\{\left(F_{d}\right)_{2 k}\right\}, \ldots, \max \left\{\left(F_{d}\right)_{m k}\right\}\right]^{T}, \\
S & =\sum_{k=1}^{q}\left[\left(1-\tau_{\omega k}\right) \zeta_{k} \widehat{\bar{\omega}}_{k}+\tau_{\omega k} \zeta_{k} \underline{\widehat{\omega}}_{k}\right],
\end{aligned}
$$

where $d=1,2, \ldots, M, P_{d}$ is a positive symmetric matrix, and $\zeta_{k}$ is the $k^{\text {th }}$ column of $\zeta=\left[\zeta_{1}, \zeta_{2}, \ldots, \zeta_{k}\right]$. Letting

$$
\begin{aligned}
& \tilde{\rho}(t)=\widehat{\rho}(t)-\rho, \\
& \tilde{\bar{\omega}}(t)=\widehat{\bar{\omega}}(t)-\bar{\omega}, \\
& \tilde{\omega}(t)=\underline{\omega}(t)-\underline{\omega},
\end{aligned}
$$

where $\rho=\operatorname{diag}\left\{\rho_{m}\right\}, \bar{\omega}=\left[\bar{\omega}_{1}, \ldots, \bar{\omega}_{q}\right]^{T}, \underline{\omega}=\left[\underline{\omega}_{1}, \ldots, \underline{\omega}_{q}\right]^{T}$, the error system can be rewritten as

$$
\begin{aligned}
\dot{\tilde{\bar{\omega}}}_{k}(t) & =\dot{\hat{\bar{\omega}}}_{k}(t), \\
\dot{\tilde{\omega}}_{k}(t) & =\underline{\hat{\omega}}_{k}(t), \\
\dot{\tilde{\rho}}_{h}(t) & =\dot{\hat{\rho}}_{h}(t) .
\end{aligned}
$$

For the MJSs with the asynchronization phenomenon which appears between the system modes and controller modes described in (10), the gains of the adaptive faulttolerant controller are proposed as follows:

$$
\begin{aligned}
& K_{2 \phi}=-B_{\phi}^{T} P_{\phi}, \\
& K_{3 \phi}=-\widehat{\rho}^{-1}(t) \zeta,
\end{aligned}
$$

and $K_{1 \phi}$ will be designed by the following LMIs: 


$$
\sum_{\phi=1}^{M} \mu_{i \phi}\left\{X_{\phi}^{T} A_{i}^{T}+A_{i} X_{\phi}+Y_{\phi}^{T} \rho_{0}^{T} B_{i}^{T}+B_{i} \rho_{0} Y_{\phi}\right\}+\sum_{j=1}^{N} \pi_{i j} \widehat{P}_{j}<0
$$

$$
\begin{gathered}
{\left[\begin{array}{ccc}
\Omega_{1} & Y_{\phi}^{T} & \sqrt{2} X_{\phi}^{T} \tilde{\delta} \\
* & -\lambda_{\phi} & 0 \\
* & * & -I
\end{array}\right]<0} \\
\Omega_{1}=X_{\phi}^{T} A_{i}^{T}+A_{i} X_{\phi}+Y_{\phi}^{T} \rho_{0}^{T} B_{i}+B_{i} \rho_{0} Y_{\phi}+\sum_{j=1}^{N} \pi_{i j} \widehat{P}_{j} \\
\quad+\lambda_{\phi} B_{i} \rho_{0} r r^{T} \rho_{0}^{T} B_{i}^{T},
\end{gathered}
$$

where $\widetilde{\delta}=\left[\max \left\{P_{\mathrm{d}} \operatorname{col}_{1}\left(B_{d}\right)\right\}, \ldots, \max \left\{P_{\mathrm{d}} \operatorname{col}_{m}\left(B_{d}\right)\right\}\right], d=$ $1,2, \ldots, M, \rho_{0}=\operatorname{diag}\left\{\rho_{01}, \rho_{02}, \ldots, \rho_{0 h}\right\}, \quad \rho_{0 h}=\left(\bar{\rho}_{h}+\rho_{h}\right) / 2$, $r=\operatorname{diag}\left\{r_{1}, r_{2}, \ldots, r_{h}\right\}, \quad r_{h}=\left(\bar{\rho}_{h}-\rho_{h}\right) /\left(\bar{\rho}_{h}+\rho_{h}\right), \quad{ }^{\prime} h=$ $1,2, \ldots, m . X_{\phi}$ is an appropriate dimension matrix and $X_{\phi}>0$, and $\lambda_{\phi}$ is a positive scalar. Then, we can obtain $K_{1 \phi}=Y_{\phi} X_{\phi}^{-1}$. After designing the controller gains, we can obtain the following theorem.

Theorem 1. Consider the closed-loop fault-tolerant MJS described by (4) which satisfies Assumptions 1-4. If there exists a symmetric matrix $P_{i}>0$, by utilizing the fault-tolerant controller (8) with the adaptive laws (11), (12) and the controller gains satisfy (16)-(18) and the asymptotical stability of the closed-loop system can be guaranteed.

Proof. First, it is more concise and does not occupy space for the subsequent proof process. We omit $(t)$ after the parameters. Such as $x(t)=x, \quad \tilde{\rho}_{h}(t)=\widetilde{\rho}_{h}, \quad \widetilde{\bar{\omega}}_{k}(t)=\widetilde{\bar{\omega}}_{k}$, $\underline{\widetilde{\omega}}_{k}(t)=\underline{\widetilde{\omega}}_{k}$, and $\hat{\rho}(t)=\hat{\rho}$. The Lyapunov function is defined in the form of the following equation:

$$
V(r(t), t)=x^{T} P_{i} x+\sum_{h=1}^{m} \frac{\widetilde{\rho}_{h}^{2}}{\gamma_{h} l_{h}}+\sum_{k=1}^{q} \frac{\widetilde{\bar{\omega}}_{k}^{2}}{\eta_{k}}+\sum_{k=1}^{q} \frac{\widetilde{\omega}_{k}^{2}}{\eta_{k}} .
$$

Let $\mathcal{U}$ be the weak infinitesimal differential operator of the stochastic process $\{(x(t), r(t)), t \geq 0\}$ :

$$
\begin{aligned}
\mathscr{U V}(r(t), t)= & \sum_{\phi=1}^{M} \mu_{i \phi} x^{T}\left\{\widehat{\mathfrak{H}}_{i \phi}^{T} P_{i}+P_{i} \widehat{\mathfrak{ज}}_{i \phi}\right\} x+x^{T} \sum_{j=1}^{N} \pi_{i j} P_{j} x \\
& +2 \sum_{\phi=1}^{M} \mu_{i \phi} x^{T} P_{i} B_{i} \rho \widehat{\rho}^{-1} K_{2 \phi} x+2 x^{T} P_{i} B_{i} F_{i} \omega \\
& +2 \sum_{\phi=1}^{M} \mu_{i \phi} x^{T} P_{i} B_{i} \rho K_{3 \phi}\left(\widehat{\bar{\omega}}+\tau_{\omega} \underline{\widehat{\omega}}-\tau_{\omega} \widehat{\bar{\omega}}\right) \\
& +2 \sum_{k=1}^{q} \frac{\widetilde{\bar{\omega}}_{k} \dot{\bar{\omega}}_{k}}{\eta_{k}}+2 \sum_{k=1}^{q} \frac{\widetilde{\widetilde{\omega}}_{k} \frac{\dot{\tilde{\omega}}}{\eta_{k}}+2 \sum_{h=1}^{m} \frac{\tilde{\rho}_{h} \dot{\tilde{\rho}}_{h}}{\gamma_{h} l_{h}}}{}
\end{aligned}
$$

where $\widehat{\mathfrak{S}}_{i \phi}=A_{i}+B_{i} \rho K_{1 \phi}$. Combining with the adaptive laws of the disturbances (12) and (20), it can be obtained that

$$
\begin{aligned}
\mathscr{U V}(r(t), t)= & \sum_{\phi=1}^{M} \mu_{i \phi} x^{T}\left\{\widehat{\mathfrak{H}}_{i \phi}^{T} P_{i}+P_{i} \widehat{\mathfrak{ज}}_{i \phi}\right\} x+x^{T} \sum_{j=1}^{N} \pi_{i j} P_{j} x \\
& +2 \sum_{\phi=1}^{M} \mu_{i \phi} x^{T} P_{i} B_{i} \rho \widehat{\rho}^{-1} K_{2 \phi} x+2 x^{T} P_{i} B_{i} F_{i} \omega \\
& +2 \delta \sum_{k=1}^{q}\left[\left(1-\tau_{\omega k}\right) \zeta_{k} \tilde{\bar{\omega}}_{k}+\tau_{\omega k} \zeta_{k} \underline{\tilde{\omega}}_{k}\right]+2 \sum_{h=1}^{m} \frac{\tilde{\rho}_{h} \dot{\tilde{\rho}}_{h}}{\gamma_{h} l_{h}} \\
& +2 \sum_{\phi=1}^{M} \mu_{i \phi} x^{T} P_{i} B_{i} \rho K_{3 \phi}\left(\widehat{\bar{\omega}}+\tau_{\omega} \underline{\widehat{\omega}}-\tau_{\omega} \widehat{\bar{\omega}}\right) .
\end{aligned}
$$

In (22), bringing $\tau_{\omega k}$ as defined in (6) and formula (22) into formula (21), it can be obtained that

$$
\begin{aligned}
x^{T} P_{i} B_{i} F_{i} \omega \leq & x^{T} P_{i} B_{i} \sum_{k=1}^{q}\left[\left(1-\tau_{\omega k}\right) \operatorname{col}_{k}\left(F_{i}\right) \bar{\omega}_{k}\right. \\
& \left.+\tau_{\omega k} \operatorname{col}_{k}\left(F_{i}\right) \underline{\omega}_{k}\right] \leq \delta \sum_{k=1}^{q}\left[\left(1-\tau_{\omega k}\right) \zeta_{k} \bar{\omega}_{k}+\tau_{\omega k} \zeta_{k} \underline{\omega}_{k}\right] .
\end{aligned}
$$

Then, we have

$$
\begin{aligned}
\mathscr{U} V(r(t), t) \leq & \sum_{\phi=1}^{M} \mu_{i \phi} x^{T}\left\{\widehat{\mathfrak{S}}_{i \phi}^{T} P_{i}+P_{i} \widehat{\mathfrak{ज}}_{i \phi}\right\} x+x^{T} \sum_{j=1}^{N} \pi_{i j} P_{j} x \\
& +2 \sum_{\phi=1}^{M} \mu_{i \phi} x^{T} P_{i} B_{i} \rho \widehat{\rho}^{-1} K_{2 \phi} x+2 \sum_{h=1}^{m} \frac{\widetilde{\rho}_{h} \dot{\tilde{\rho}}_{h}}{\gamma_{h} l_{h}} \\
& +2 \sum_{\phi=1}^{M} \mu_{i \phi} x^{T} P_{i} B_{i} \rho K_{3 \phi}\left(\widehat{\bar{\omega}}+\tau_{\omega} \underline{\widehat{\omega}}-\tau_{\omega} \widehat{\bar{\omega}}\right) \\
& +2 \delta \sum_{k=1}^{q}\left[\left(1-\tau_{\omega k}\right) \zeta_{k} \hat{\bar{\omega}}_{k}+\tau_{\omega k} \zeta_{k} \widehat{\widehat{\omega}}_{k}\right] .
\end{aligned}
$$

Bringing the error system (14), the derivative of error system (15), and the partial of controller $K_{2 \phi}, K_{3 \phi}$ (16) into (23), the inequality can be obtained as the following form:

$$
\begin{aligned}
\mathscr{U} V(r(t), t) \leq & \sum_{\phi=1}^{M} \mu_{i \phi} x^{T}\left\{\widehat{\mathfrak{H}}_{i \phi}^{T} P_{i}+P_{i} \widehat{\mathfrak{H}}_{i \phi}\right\} x+x^{T} \sum_{j=1}^{N} \pi_{i j} P_{j} x \\
& +2 \delta \sum_{k=1}^{q}\left[\left(1-\tau_{\omega k}\right) \zeta_{k} \widehat{\bar{\omega}}_{k}+\tau_{\omega k} \zeta_{k} \widehat{\omega}_{k}\right]+2 \sum_{h=1}^{m} \frac{\widetilde{\rho}_{h} \dot{\tilde{\rho}}_{h}}{\gamma_{h} l_{h}} \\
& -2 \sum_{\phi=1}^{M}\left\{\mu_{i \phi} \delta \rho \widehat{\rho}^{-1} \sum_{k=1}^{q}\left[\left(1-\tau_{\omega k}\right) \zeta_{k} \widehat{\bar{\omega}}_{k}+\tau_{\omega k} \zeta_{k} \widehat{\underline{\omega}}_{k}\right]\right\} \\
& +2 \sum_{\phi=1}^{M} \mu_{i \phi} x^{T} P_{i} B_{i}(\widehat{\rho}-\tilde{\rho}) \widehat{\rho}^{-1}\left(-B_{\phi}^{T} P_{\phi}\right) x .
\end{aligned}
$$


Based on (13), the inequality can be rewritten as

$$
\begin{aligned}
\mathscr{U V}(r(t), t) \leq & \sum_{\phi=1}^{M} \mu_{i \phi} x^{T}\left\{\widehat{\mathfrak{H}}_{i \phi}^{T} P_{i}+P_{i} \widehat{\mathfrak{S}}_{i \phi}\right\} x+x^{T} \sum_{j=1}^{N} \pi_{i j} P_{j} x \\
& +2 \sum_{\phi=1}^{M}\left\{\mu_{i \phi} \delta \widetilde{\rho} \widehat{\rho}^{-1} \sum_{k=1}^{q}\left[\left(1-\tau_{\omega k}\right) \zeta_{k} \widehat{\bar{\omega}}_{k}+\tau_{\omega k} \zeta_{k} \widehat{\omega}_{k}\right]\right\} \\
& +\sum_{\phi=1}^{M} \mu_{i \phi} x^{T} P_{i} B_{i}(\widehat{\rho}-\widetilde{\rho}) \widehat{\rho}^{-1}\left(B_{i}^{T} P_{i}\right) x \\
& +\sum_{\phi=1}^{M} \mu_{i \phi} x^{T} P_{\phi} B_{\phi}(\widehat{\rho}-\widetilde{\rho}) \widehat{\rho}^{-1}\left(B_{\phi}^{T} P_{\phi}\right) x+2 \sum_{h=1}^{m} \frac{\tilde{\rho}_{h} \dot{\tilde{\rho}}_{h}}{\gamma_{h} l_{h}} \\
\leq & \sum_{\phi=1}^{M} \mu_{i \phi} x^{T}\left\{\widehat{\mathfrak{H}}_{i \phi}^{T} P_{i}+P_{i} \widehat{\mathfrak{S}}_{i \phi}\right\} x+x^{T} \sum_{j=1}^{N} \pi_{i j} P_{j} x \\
& +2 \sum_{\phi=1}^{M} \mu_{i \phi}\left[\sum_{h=1}^{m} \widetilde{\rho}_{h} \widehat{\rho}_{h}^{-1} \delta_{h} S_{h}\right]+2 \sum_{h=1}^{m} \frac{\tilde{\rho}_{h} \dot{\tilde{\rho}}_{h}}{\gamma_{h} l_{h}} \\
& -2 \sum_{\phi=1}^{M} \mu_{i \phi}\left[\sum_{h=1}^{m} \widetilde{\rho}_{h} \widehat{\rho}_{h}^{-1} \delta_{h}^{2}\right]+2 \sum_{\phi=1}^{M} \mu_{i \phi} \delta \delta^{T} .
\end{aligned}
$$

Note that the adaptive law of the failure $\rho_{h}$ is chosen as (11), and then (25) can be rewritten as

$$
\begin{aligned}
\mathcal{U V}(r(t), t) \leq & \sum_{\phi=1}^{M} \mu_{i \phi} x^{T}\left\{\widehat{\mathfrak{H}}_{i \phi}^{T} P_{i}+P_{i} \widehat{\mathfrak{H}}_{i \phi}\right\} x+x^{T} \sum_{j=1}^{N} \pi_{i j} P_{j} x \\
& +2 \sum_{\phi=1}^{M} \mu_{i \phi} \delta \delta^{T} .
\end{aligned}
$$

For any $\rho$, the $K_{1 \phi}$ will be designed by the following inequality:

$$
\begin{aligned}
& \sum_{\phi=1}^{M} \mu_{i \phi}\left\{X_{\phi}^{T} A_{i}^{T}+A_{i} X_{\phi}\right\}+\sum_{j=1}^{N} \pi_{i j} \widehat{P}_{j} \\
& \quad+\sum_{\phi=1}^{M} \mu_{i \phi}\left\{Y_{\phi}^{T} \rho^{T} B_{i}^{T}+B_{i} \rho Y_{\phi}\right\}+2 \sum_{\phi=1}^{M} \mu_{i \phi} X_{\phi}^{T} \widetilde{\delta} \widetilde{\delta}^{T} X_{\phi}<0 .
\end{aligned}
$$

However, the exact values of $\rho$ is not known in advance and the upper and lower bounds of $\rho$ is used to design the controller gain $K_{1 \phi}$; by denoting

$$
\begin{gathered}
\rho_{0 h}=\frac{\underline{\rho}_{h}+\bar{\rho}_{h}}{2}, \\
g_{h}=\frac{\rho_{h}-\rho_{0 h}}{\rho_{0 h}}, \\
r_{h}=\frac{\bar{\rho}_{h}-\underline{\rho}_{h}}{\bar{\rho}_{h}+\underline{\rho}_{h}},
\end{gathered}
$$

we have

$$
\rho_{h}=\rho_{0 h}\left(1+g_{h}\right), \quad\left|g_{h}\right| \leq r_{h}<1, h=1,2, \ldots, m .
$$

Define

$$
\begin{aligned}
r & =\operatorname{diag}\left\{r_{1}, r_{2}, \ldots, r_{m}\right\}, \\
\rho_{0} & =\operatorname{diag}\left\{\rho_{01}, \rho_{02}, \ldots, \rho_{0 m}\right\}, \\
g & =\operatorname{diag}\left\{g_{1}, g_{2}, \ldots, g_{m}\right\}, \\
|g| & =\operatorname{diag}\left\{\left|g_{1}\right|,\left|g_{2}\right|, \ldots,\left|g_{m}\right|\right\},
\end{aligned}
$$

and the failure matrix $\rho$ can be described as

$$
\begin{aligned}
\rho=\rho_{0}(I+g) & \\
& |g| \leq r<I .
\end{aligned}
$$

Considering both (27) and (31), inequality (27) can be written as

$$
\begin{aligned}
& \sum_{\phi=1}^{M} \mu_{i \phi}\left\{X_{\phi}^{T} A_{i}^{T}+A_{i} X_{\phi}\right\}+\sum_{j=1}^{N} \pi_{i j} \widehat{P}_{j} \\
& \quad+\sum_{\phi=1}^{M} \mu_{i \phi}\left\{Y_{\phi}^{T} \rho_{0}^{T} B_{i}^{T}+B_{i} \rho_{0} Y_{\phi}\right\} \\
& \quad+\sum_{\phi=1}^{M} \mu_{i \phi}\left\{Y_{\phi}^{T} g^{T} \rho_{0}^{T} B_{i}^{T}+B_{i} \rho_{0} g Y_{\phi}\right\}+2 \sum_{\phi=1}^{M} \mu_{i \phi} X_{\phi}^{T} \widetilde{\delta} \widetilde{\delta}^{T} X_{\phi}<0 .
\end{aligned}
$$

By using the Schur complement, the corresponding results in (17) and (18) can be obtained directly. Hence, it is easy to find that $\mathscr{U} V(r(t), t)<0$ for any $x \neq 0$. Thus, the solutions of the asynchronous closed-loop FTC system are uniformly bounded and the states $x(t)$ converge asymptotically to 0 . This is the end of the proof.

\section{Numerical Example}

In this section, two numerical examples are given to verify the validity of our method.

Example 1. The closed-loop system is considered as the following:

$$
\begin{aligned}
\dot{x}(t)= & \left(A_{i}+B_{i} \rho K_{1 \phi}+B_{i} \rho \widehat{\rho}^{-1}(t) K_{2 \phi}\right) x(t)+B_{i} F_{i} \omega(t) \\
& +B_{i} \rho K_{3 \phi}\left[\left(I-\tau_{\omega}\right) \hat{\bar{\omega}}(t)+\tau_{\omega} \underline{\widehat{\omega}}(t)\right] .
\end{aligned}
$$

Considering the system with three operating modes, the parameters of the three system modes are selected as 


$$
\begin{aligned}
& A_{1}=\left[\begin{array}{ccc}
-2.4 & 2 & 3 \\
-1.3 & -0.8 & 1.8 \\
4 & 0.3 & -1.3
\end{array}\right] \text {, } \\
& A_{2}=\left[\begin{array}{ccc}
-2.9 & 2 & 0 \\
-0.7 & -0.3 & 2 \\
2 & 0.5 & -1.7
\end{array}\right] \text {, } \\
& A_{3}=\left[\begin{array}{ccc}
-1.9 & 0 & 2 \\
-1.7 & -1.3 & 2 \\
2 & 0.5 & -1.7
\end{array}\right] \text {, } \\
& B_{1}=\left[\begin{array}{ccc}
1 & 0.4 & 1.1 \\
3.5 & 3 & 0
\end{array}\right]^{T} \text {, } \\
& B_{2}=\left[\begin{array}{ccc}
1 & 0.5 & 0 \\
2.1 & 2.9 & 1
\end{array}\right]^{T} \text {, } \\
& B_{3}=\left[\begin{array}{ccc}
1 & 0.8 & 1 \\
0 & 3 & 2.1
\end{array}\right]^{T} \text {, } \\
& F_{1}=\left[\begin{array}{ccc}
1 & 0.4 & 0.3 \\
-1 & -0.9 & 0.2
\end{array}\right] \text {, } \\
& F_{2}=\left[\begin{array}{ccc}
0.9 & 0.6 & 2 \\
-0.2 & -0.7 & 1
\end{array}\right] \text {, } \\
& F_{3}=\left[\begin{array}{ccc}
1.2 & 0.7 & 3 \\
0 & -0.5 & 3
\end{array}\right] \text {. }
\end{aligned}
$$

The transition rate matrix of system modes $i \in\{1,2,3\}$ and controller modes $\phi \in\{1,2,3\}$ is given as follows:

$$
\begin{aligned}
& \boldsymbol{\Pi}=\left[\begin{array}{ccc}
-0.8 & 0.4 & 0.4 \\
0.3 & -0.6 & 0.3 \\
0.25 & 0.25 & -0.5
\end{array}\right], \\
& \boldsymbol{\Omega}=\left[\begin{array}{lll}
0.3 & 0.3 & 0.4 \\
0.2 & 0.5 & 0.3 \\
0.4 & 0.4 & 0.2
\end{array}\right] .
\end{aligned}
$$

To illustrate the effectiveness of our method for those systems, the following initial conditions and parameters of the simulation are given as $\widehat{\rho}_{h}(0)=0.7, \widehat{\bar{\omega}}_{k}(0)=0.4, \underline{\widehat{\omega}}_{k}(0)=$ $-0.3, h=1,2, k=1,2,3, x_{1}(0)=70, x_{2}(0)=35, x_{3}(0)=$ $-75, l_{1}=0.5, l_{2}=0.2, \eta_{1}=0.2, \eta_{2}=0.5, \eta_{3}=0.3$. The following failure condition is considered in the simulations: before 60 seconds, the system is running with the disturbances $\omega(t)=[-0.02 \sin \quad(0.1 t)-0.25,0.03 \sin (0.3 t)$, $\left.e^{-0.5 t} \sin (0.07 t)-0.1 \sin \left(0.01 x_{1}(t)\right)+0.25\right]^{T}$, and there is no actuator failure which means $\rho=\operatorname{diag}[1,1]$. At $60 \mathrm{sec}-$ onds, some failures in the actuators have occurred, described by $\rho=\operatorname{diag}[0.4,0.6]$.

The states of the open-loop system are shown in Figure 1, and it can be found that the states of the system are divergent, so the open-loop system is an unstable system. When the system is running without failure, the states of that system are shown in Figure 2. This figure illustrates that the states of this closed-loop system are converged to 0 . The states of the closed-loop system which have occurred failure are shown in Figure 3, and it is obvious that the trend of the states begin to diverge after the failure occurred for a while but finally they converged to 0 under the effect of the controller. The estimations of the actuator failures and disturbances are shown in Figures 4 and 5. Hence, this simple example illustrates that the controller designed by our method can compensate the effects from the actuator failures and disturbances well under the asynchronization phenomenon between the system modes and controller modes.

Example 2. In this example, we consider a single link robot arm system in [33] to verify our method and it is given as follows:

$$
\ddot{\theta}(t)=-\frac{M_{i} g L}{J_{i}} \sin (\theta(t))-\frac{D}{J_{i}} \dot{\theta}(t)+\frac{1}{J_{i}}\left[u(t)+d_{1}(t)\right]+\frac{1}{J_{i}} d_{2}(t),
$$

where $\theta(t)$ is the angle position of the robot arm, $u(t)$ is the control input, $d_{1}(t)$ and $d_{2}(t)$ are the exogenous disturbances, $M_{i}$ is the mass of the payload, $g$ is the acceleration of gravity, $L$ is the length of the arm, $J_{i}$ is the moment of inertia, and $D$ is the viscous friction. The parameters are assumed as $g=9.8, L=0.04$, and $D=2$. Defining that $x_{1}(t)=\theta(t)$ and $x_{2}(t)=\dot{\theta}(t)$, system (36) can be rewritten as

$$
\begin{aligned}
\dot{x}(t)= & {\left[\begin{array}{cc}
0 & 1 \\
0 & -\frac{D}{J_{i}}
\end{array}\right] x(t)+\left[\begin{array}{c}
0 \\
1 \\
J_{i}
\end{array}\right] u(t) } \\
& +\left[\begin{array}{cc}
0 & 0 \\
\frac{1}{J_{i}} & \frac{1}{J_{i}}
\end{array}\right]\left[\begin{array}{c}
d_{1} \\
d_{2}-M_{i} g L \sin \left(x_{1}(t)\right)
\end{array}\right] .
\end{aligned}
$$

The parameters of the modes are given as follows:

$$
\begin{array}{r}
i \in\{1,2,3,4\}, \\
J_{1}=1.0, \\
J_{2}=1.2, \\
J_{3}=2.0, \\
J_{4}=1.5, \\
\phi \in\{1,2,3,4\}, \\
M_{1}=1.0, \\
M_{2}=0.4, \\
M_{3}=1.0, \\
M_{4}=0.5 .
\end{array}
$$

The system transition rate matrix $\Pi$ and the controller transition matrix $\boldsymbol{\Omega}$ are presented as 


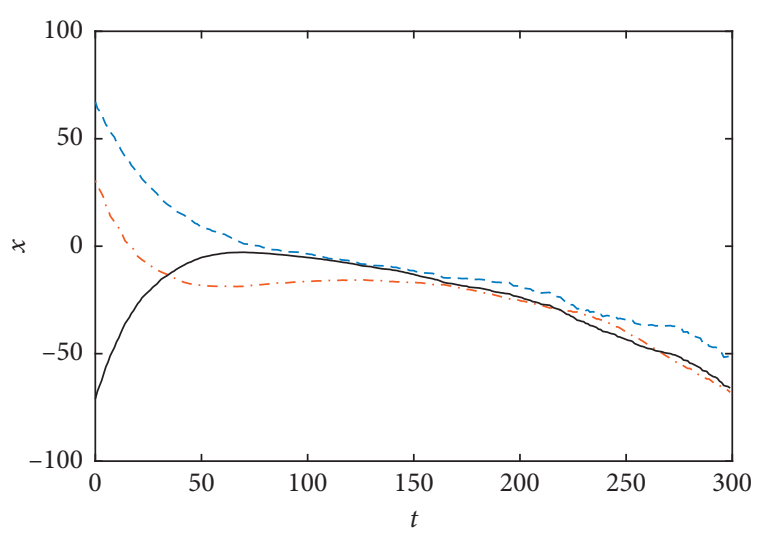

$--x_{1}$

$\cdot-\cdot-x_{2}$

$-x_{3}$

FIGURE 1: The states of the open-loop system.

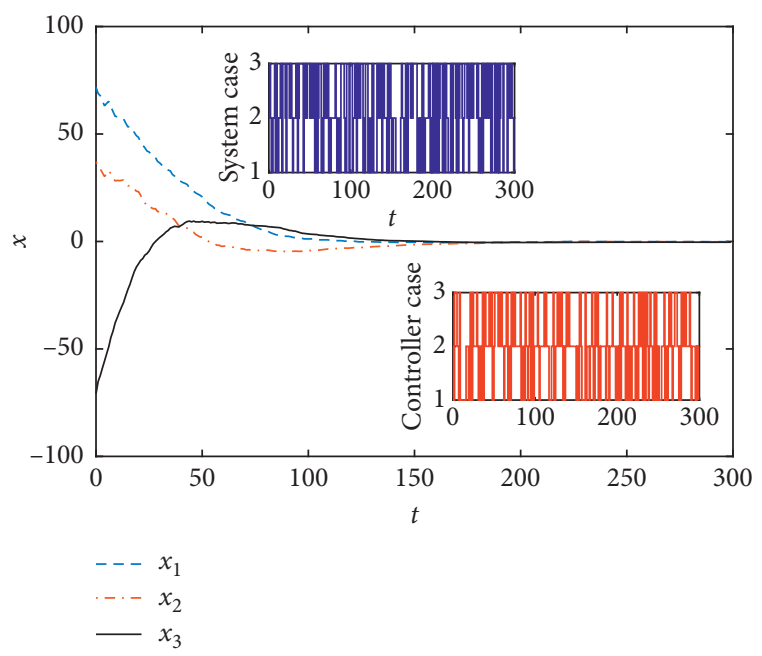

FIgURE 2: The states of the closed-loop system without failure.

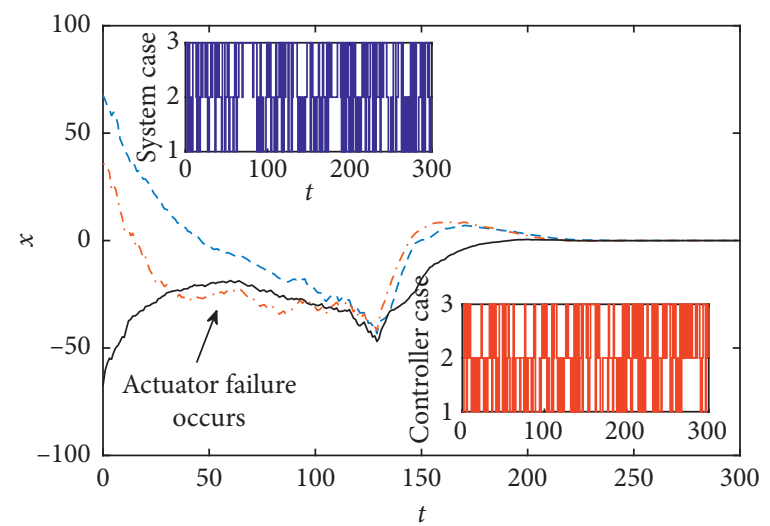

$$
\begin{aligned}
---x_{1} \\
-\cdot-x_{2} \\
-\quad x_{3}
\end{aligned}
$$

FIgURE 3: The states of the closed-loop system with failure.

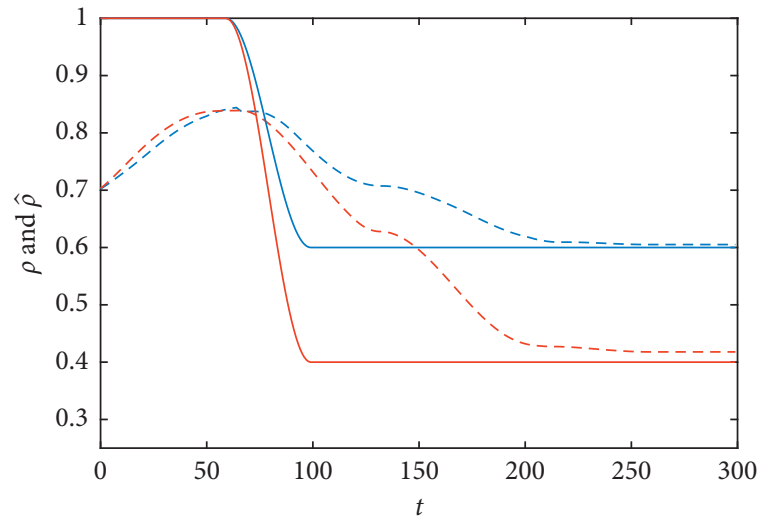

$$
\begin{array}{ll}
--\hat{\rho}_{2} & -\hat{\rho}_{2} \\
---\hat{\rho}_{1} & -\hat{\rho}_{1}
\end{array}
$$

FIgURE 4: The estimations of actuator failure factor $\rho$.

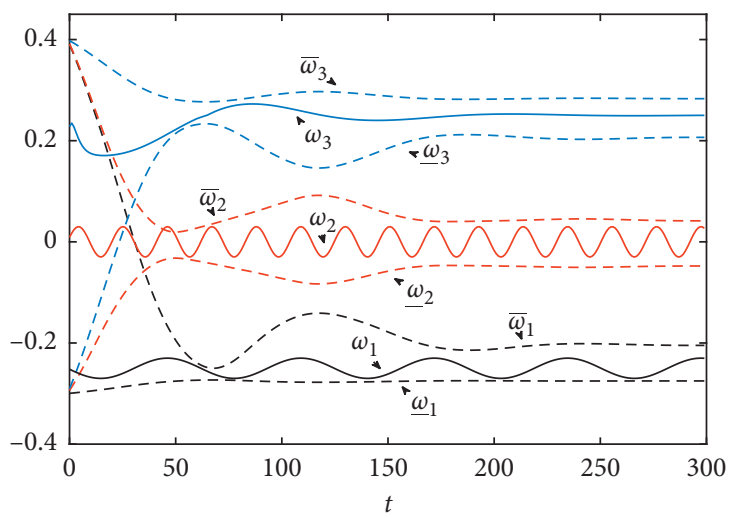

FIGURE 5: The estimations of upper bound and lower bound of $\omega$.

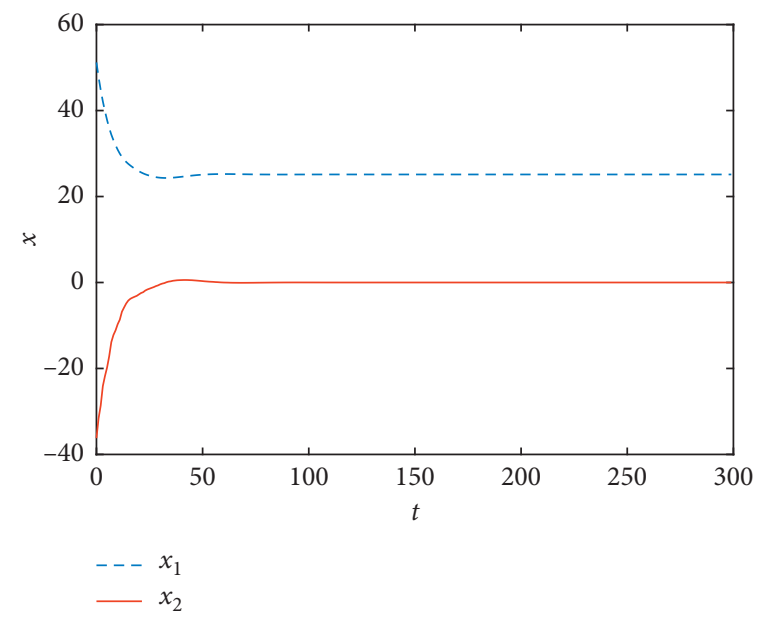

Figure 6: The states of the open-loop system. 


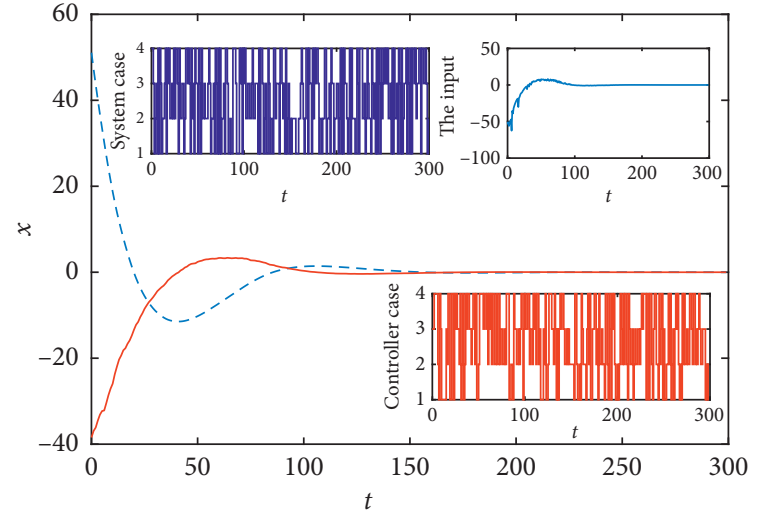

$--x_{1}$

$-x_{2}$

FIgURE 7: The states of the closed-loop system without failure.

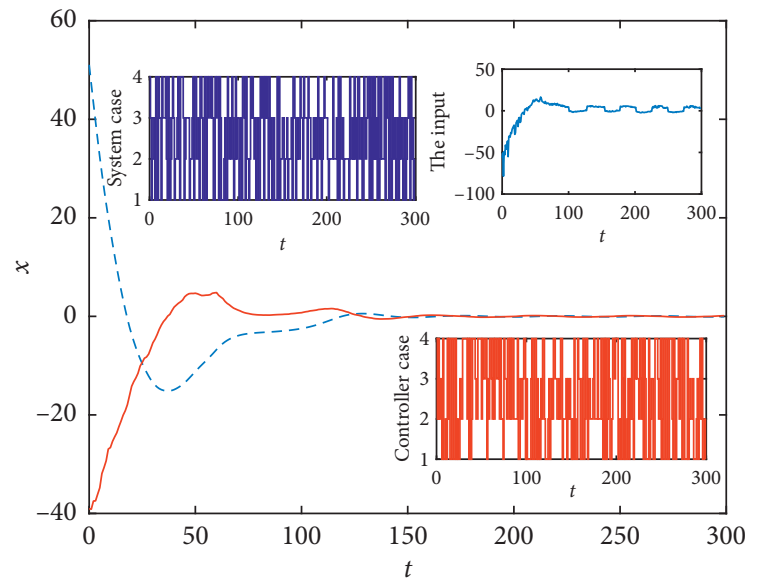

$--x_{1}$

$-x_{2}$

Figure 8: The states of the closed-loop system with failure.

$$
\begin{aligned}
& \Pi=\left[\begin{array}{cccc}
-0.9 & 0.4 & 0.4 & 0.1 \\
0.1 & -0.6 & 0.4 & 0.1 \\
0.5 & 0.1 & -0.7 & 0.1 \\
0.2 & 0.2 & 0.4 & -0.8
\end{array}\right], \\
& \boldsymbol{\Omega}=\left[\begin{array}{llll}
0.3 & 0.5 & 0.1 & 0.1 \\
0.2 & 0.2 & 0.2 & 0.4 \\
0.3 & 0.2 & 0.1 & 0.4 \\
0.1 & 0.1 & 0.4 & 0.4
\end{array}\right]
\end{aligned}
$$

The simulations are given with the following initial conditions and parameters as follows:

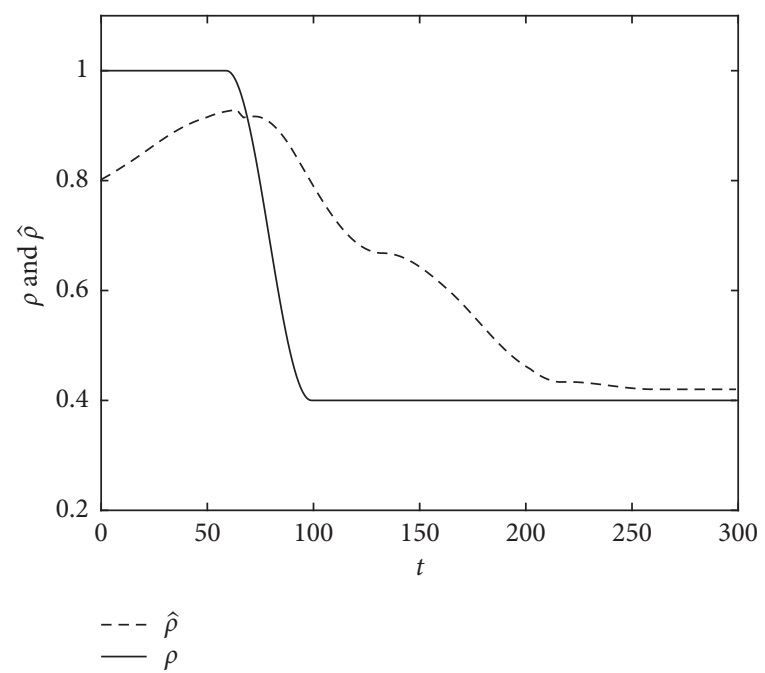

FIgURE 9: The estimation of the actuator failure factor $\rho$.

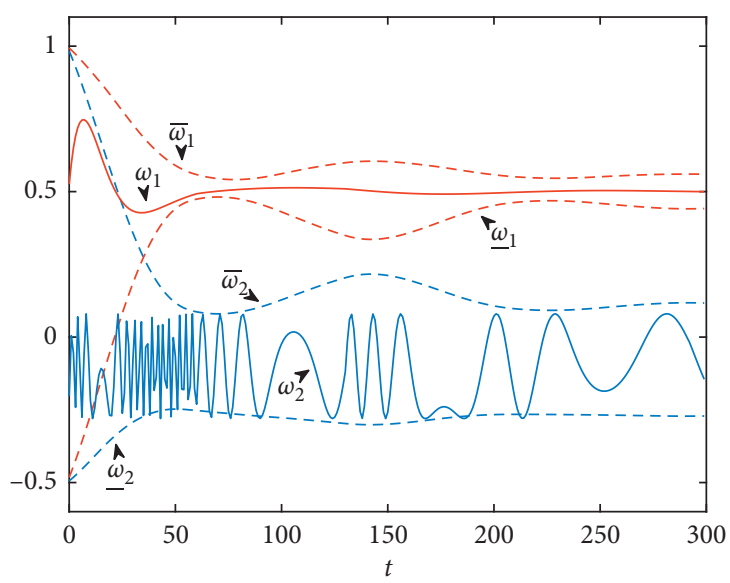

Figure 10: The estimations of upper bound and lower bound of $\omega$.

$$
\begin{aligned}
\widehat{\rho}_{1}(0) & =0.8, \\
\widehat{\bar{\omega}}_{k}(0) & =1, \\
\widehat{\underline{\omega}}_{k}(0) & =-0.5, \\
k & =1,2, \\
x_{1}(0) & =55, \\
x_{2}(0) & =-40, \\
l_{1} & =0.4, \\
\eta_{1} & =0.3, \\
\eta_{2} & =0.7 .
\end{aligned}
$$

The failure condition is considered in this simulation: in the first 60 seconds, the system is running with the disturbances

$$
\omega(t)=\left[e^{-0.1 t} \sin (0.1 t)-0.1 \sin \left(0.01 x_{1}(t)\right)+0.5, e^{-t} \sin (0.1 t)-M g L \sin \left(0.01 x_{1}\right)-0.1\right]^{T},
$$




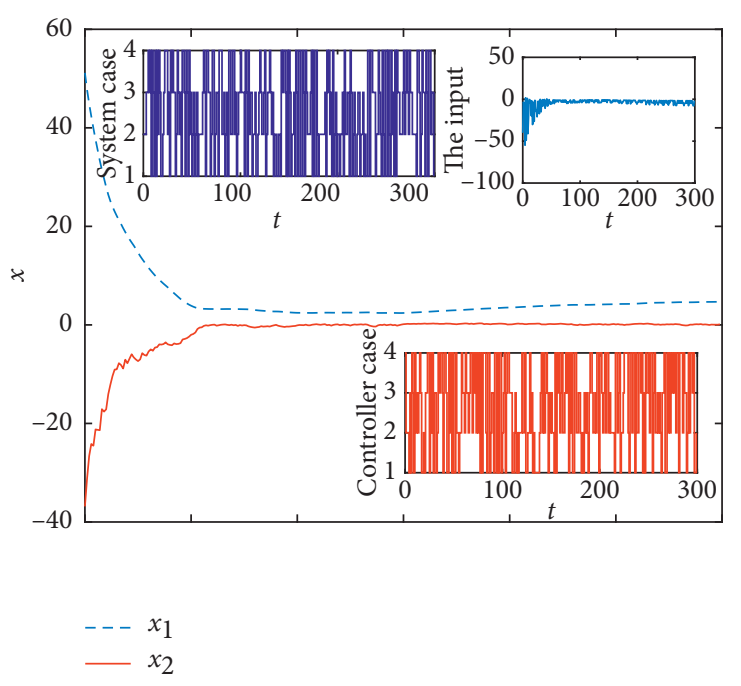

Figure 11: The states of the method in reference [33].

and there is no actuator failure which means $\rho=1$. Starting from 65 seconds, partial failure of the actuator has occurred, described by $\rho=0.4$.

The states of the open-loop system are presented in Figure 6, and from this figure it can be found that the states of the system are convergent but they are not converged to 0 , which means that the open-loop system is just a stable system but not asymptotically stable. So, this system is necessary to introduce the controller to improve the performance of the overall system. We firstly consider the system running without failure, and the states of that system are presented in Figure 7. It can be found that the states of this closed-loop system converge to 0 , while the controller is introduced into the open-loop system. After this condition, we consider that partial failure of the actuator has occurred when the system is running after a few seconds. The final result is presented in Figure 8, and even if the actuator failure occurred (Figure 9) and time-varying disturbances exist (Figure 10), the states of the closed-loop system which have been introduced into the controller we have designed will eventually converge to 0 . By using the algorithm in [33], the state trajectory of the closedloop is shown in Figure 11. It can be found that when there is an asynchronization phenomenon, the method in reference [33] does not converge all the states of the system to 0 . Thus, the controller designed by our method can achieve better control effects in the above condition.

These two numerical examples illustrate that the controller designed by our method can compensate the effects from the actuator failures and disturbances well for the MJSs with the asynchronization phenomenon.

\section{Conclusion}

In this paper, an HMM-based asynchronous fault-tolerant controller is provided for MJSs with actuator failures and unknown nonlinear disturbances. By designing suitable adaptive laws, the values of partial failures and the bounds of disturbances are estimated, respectively. Based on the provided adaptive laws, a kind of asynchronous adaptive fault-tolerant controller algorithm is given, where part of the controller gains are obtained via LMI technology. Finally, the effectiveness of the proposed method is illustrated with two numerical examples.

\section{Data Availability}

The data supporting this research are from previously reported studies and datasets, which have been cited. The processed data are available from reference [33] in the article.

\section{Conflicts of Interest}

The authors declare that they have no conflicts of interest.

\section{Acknowledgments}

This work was supported by the Zhejiang Provincial Natural Science Foundation of China (no. LY19F030020), National Natural Science Foundation of China (no. 61733009), and NSFC-Zhejiang Joint Fund for the Integration of Industrialization and Informatization (nos. U1709215 and U1509203).

\section{References}

[1] J.-N. Li, Y.-F. Xu, K.-Y. Gu, L.-S. Li, and X.-B. Xu, "Mixed passive $/ H_{\infty}$ hybrid control for delayed Markovian jump system with actuator constraints and fault alarm," International Journal of Robust and Nonlinear Control, vol. 28, no. 18, pp. 6016-6037, 2018.

[2] P. Bolzern, P. Colaneri, and G. De Nicolao, "Stochastic stability of positive Markov jump linear systems," Automatica, vol. 50, no. 4, pp. 1181-1187, 2014.

[3] Y. Ji and H. J. Chizeck, "Controllability, stabilizability, and continuous-time Markovian jump linear quadratic control," IEEE Transactions on Automatic Control, vol. 35, no. 7, pp. 777-788, 1990.

[4] Y. Fang and K. A. Loparo, "Stabilization of continuous-time jump linear systems," IEEE Transactions on Automatic Control, vol. 47, pp. 1590-1603, 2002.

[5] H. Huang, F. Long, and C. Li, "Stabilization for a class of Markovian jump linear systems with linear fractional uncertainties," International Journal of Innovative Computing Information and Control, vol. 11, pp. 295-307, 2015.

[6] M. Sathishkumar, R. Sakthivel, F. Alzahrani, B. Kaviarasan, and Y. Ren, "Mixed $H_{\infty}$ and passivity-based resilient controller for nonhomogeneous Markov jump systems," Nonlinear Analysis: Hybrid Systems, vol. 31, pp. 86-99, 2019.

[7] E. K. Boukas, Z. K. Liu, and F. Al-Sunni, "Guaranteed cost control of a Markov jump linear uncertain system using a time-multiplied cost function," Journal of Optimization Theory and Applications, vol. 116, no. 1, pp. 183-204, 2003.

[8] R. Sakthivel, A. Parivallal, B. Kaviarasan, H. Lee, and Y. Lim, "Finite-time consensus of Markov jumping multi-agent systems with time-varying actuator faults and input saturation," ISA Transactions, vol. 83, pp. 89-99, 2018.

[9] K. Liang, M. Dai, H. Shen, J. Wang, Z. Wang, and B. Chen, " $L_{2}-L_{\infty}$ synchronization for singularly perturbed complex networks with semi-Markov jump topology," Applied Mathematics and Computation, vol. 321, pp. 450-462, 2018. 
[10] R. Sakthivel, R. Sakthivel, V. Nithya, P. Selvaraj, and O. M. Kwon, "Fuzzy sliding mode control design of Markovian jump systems with time-varying delay," Journal of the Franklin Institute, vol. 355, no. 14, pp. 6353-6370, 2018.

[11] Z.-G. Wu, P. Shi, H. Su, and J. Chu, "Asynchronous $L_{2}-L_{\infty}$ filtering for discrete-time stochastic Markov jump systems with randomly occurred sensor nonlinearities," Automatica, vol. 50, no. 1, pp. 180-186, 2014.

[12] Y. Zhu, L. Zhang, and W. X. Zheng, "Distributed $H_{\infty}$ filtering for a class of discrete-time Markov jump Lur'e systems with redundant channels," IEEE Transactions on Industrial Electronics, vol. 63, pp. 1876-1885, 2016.

[13] S. Dong, Z. Wu, H. Su et al., "Asynchronous control of continuous-time nonlinear Markov jump systems subject to strict dissipativity," IEEE Transactions on Automatic Control, vol. 64, pp. 1250-1256, 2018.

[14] Z. Wu, P. Shi, Z. Shu et al., "Passivity-based asynchronous control for Markov jump systems," IEEE Transactions on Automatic Control, vol. 62, pp. 2020-2025, 2017.

[15] Z. Xiang and Q. Chen, "Robust reliable control for uncertain switched nonlinear systems with time delay under asynchronous switching," Applied Mathematics and Computation, vol. 216, pp. 800-811, 2010.

[16] M. T. Raza, A. Q. Khan, G. Mustafa et al., "Design of fault detection and isolation filter for switched control systems under asynchronous switching," IEEE Transactions on Industrial Electronics, vol. 24, pp. 13-23, 2016.

[17] J. Li, Y. J. Pan, H. Su et al., "Stochastic reliable control of a class of networked control systems with actuator faults and input saturation," International Journal of Control, Automation and Systems, vol. 12, pp. 564-571, 2014.

[18] J. Li and L. Li, "Reliable control for bilateral teleoperation systems with actuator faults using fuzzy disturbance observer," IET Control Theory and Applications, vol. 11, pp. 446-455, 2016.

[19] J. Li, W. Bao, S. Li et al., "Exponential synchronization of discrete-time mixed delay neural networks with actuator constraints and stochastic missing data," Neurocomputing, vol. 207, pp. 700-707, 2016.

[20] J. Li and W. Ren, "Finite-horizon $H_{\infty}$ fault-tolerant constained consensus for multiagent systems with communication delays," IEEE Transactions on Cybernetics, 2020.

[21] B. Jiang and F. N. Chowdhury, "Fault estimation and accommodation for linear MIMO discrete-time systems," IEEE Transactions Control System Technology, vol. 13, pp. 493-499, 2005.

[22] W. Chen and F. N. Chowdhury, "Analysis and detection of incipient faults in post-fault systems subject to adaptive faulttolerant control," International Journal of Adaptive Control and Signal Processing, vol. 22, pp. 815-832, 2008.

[23] X. Li and G. Yang, "Robust adaptive fault-tolerant control for uncertain linear systems with actuator failures," IET Control Theory and Applications, vol. 6, pp. 1544-1551, 2012.

[24] L. Wu, G. Yang, and D. Ye, "Robust adaptive fault-tolerant control for linear systems with actuator failures and mismatched parameter uncertainties," IET Control Theory and Applications, vol. 8, pp. 441-449, 2014.

[25] P. A. Ioannou and P. V. Kokotovic, "Instability analysis and improvement of robustness of adaptive control," Automatica, vol. 20, pp. 583-594, 1984.

[26] X. Tang, G. Tao, and S. M. Joshi, "Adaptive actuator failure compensation for nonlinear MIMO systems with an aircraft control application," Automatica, vol. 43, pp. 1869-1883, 2007.
[27] G. Tao, S. M. Joshi, and X. Ma, "Adaptive state feedback and tracking control of systems with actuator failures," IEEE Transactions on Automatic Control, vol. 46, pp. 78-95, 2001.

[28] M. Liu, D. W. Ho, and P. Shi, "Adaptive fault-tolerant compensation control for Markovian jump systems with mismatched external disturbance," Automatica, vol. 58, pp. 5-14, 2015.

[29] Y. Xia, M. Fu, P. Shi et al., "Adaptive backstepping controller design for stochastic jump systems," IEEE Transactions on Automatic Control, vol. 54, pp. 2853-2859, 2009.

[30] M. Li, M. Liu, and Y. Zhang, "Asynchronous adaptive quantized feedback sliding mode control for semi-markovian jump systems: an event-triggered approach," Nonlinear Analysis: Hybrid System, vol. 36, Article ID 100853, 2020.

[31] M. Li, M. Liu, and Y. Zhang, "Asynchronous adaptive dynamic output feedback sliding mode control for singular markovian jump systems with actuator faults and uncertain transition rates," Applied Mathematics and Computation, vol. 371, Article ID 124958, 2020.

[32] L. R. Rabiner, "A tutorial on hidden Markov models and selected applications in speech recognition," Proceedings of IEEE, vol. 77, pp. 257-286, 1989.

[33] Y. Li, H. Sun, G. Zong et al., "Disturbance-observer-basedcontrol and $L_{2}-L_{\infty}$ resilient control for Markovian jump non-linear systems with multiple disturbances and its application to single robot arm system," IET Control Theory and Applications, vol. 10, pp. 226-233, 2016. 